MARIA.

\title{
I. FREQUÊNCIA DE NASCIMENTOS GEMELARES NO HOSPITAL DA UNIVERSIDADE DE SANTA MARIA NO PERÍODO DE 1985 A 1995
}

Claudete Maria Bittencourt de Borba Brambilla, Sílvia Alix Correa-Costa, Eduardo Militz da Costa e Liane Rosso Giuliani

Departamento de Biologia - CCNE

UFSM - Santa Maria - RS

\section{RESUMO}

Nesse trabalho procurou-se estabelecer a freqüência de nascimentos gemelares no Hospital da Universidade de Santa Maria (HUSM), no período 1985-1995, quando ocorreram 253 gestações duplas ( $1,58 \%$ ) e 6 gestações triplas ( $0,037 \%)$, num total de 16006 partos. Através do método de Weinberg, corrigido por James,foi estabelecida uma frequência de $44,75 \%$ de pares dizigóticos e, portanto, de $55,25 \%$ de pares monozigóticos. A análise da razão de sexo mostrou, entre os gêmeos, 85,82 crianças do sexo masculino por 100 do sexo feminino, resultado que diferiu significativamente do encontrado nos partos únicos, onde ocorreram 103,89 do sexo masculino por 100 do sexo feminino. Tais resultados foram comparados com os encontrados em outras populações. 


\section{SUMMARY}

FREQUENCY OF TWIN BIRTHS IN SANTA MARIA-FREQUENCY OF TWIN BIRTHS AT THE UNIVERSITY HOSPITAL IN THE PERIOD OF 1985-1995.

The frequency of twin births was established at Santa Maria's University Hospital in the period from 1985 through 1995, when 253 double gestations $(1,58 \%)$ and 6 triple gestations $(0,037 \%)$ occurred, in a total of 16006 pregnancies.With the use of Weimberg's method, corrected by James, a frequency of $44,75 \%$ of dizygotic and therefore $55,25 \%$ of monozygotic twins pairs was found. The sex ratio showed that there were 85,82 males to 100 females among the twins, result that differed significantly of the sex ratio in single pregnancies, where 103,89 males to 100 females occurred.These results are compared with those found in other populations.

\section{INTRODUÇÃO}

O estudo de pares de gêmeos tem sido tradicionalmente empregado em diferentes áreas de pesquisa de Genética Humana, com a finalidade de averiguar a influência relativa do genótipo e do ambiente sobre a variação fenotípica normal ou patológica. (BEIGUELMAN,1994).

A incidência de gêmeos nas populações humanas mostra grandes variações, com valores que vão de 5,9 por mil recém nascidos no Japão (IMAIZUMI \& INOUYE, 1979), até 52 por mil nascimentos na Nigéria (NYLANDER, 1970),ficando as populações caucasóides com valores intermediários em torno de 8 por mil (BEIGUELMAN, 1994). Tais diferenças dependem basicamente da freqüência de nascimentos de gêmeos dizigóticos ( $D Z$ ), pois a incidência de gêmeos monozigóticos ( $M Z$ ) é relativamente constante e em torno de 3 a 4 por mil recém-nascidos. (PARISI \& CARPENA, 1981).

A probabilidade de nascimento de gêmeos dizigóticos aumenta com a idade materna, como sugerido por WEINBERG em 1901. Trabalhos 
subsequentes confirmaram essa indicação, mostrando que a taxa de gêmeos $D Z$ aumenta de $O$ (zero) na puberdade até uma taxa de $0,7 \%-0,8 \%$ aos 35-39 anos, declinando posteriormente. (McARTUR, 1953; PROPPING \& VOGEL,1976 ). VOGEL \& MOTULSKY( 1986) apontam, como fator de aumento da taxa de gêmeos $D Z$ não apenas a idade materna, como também a ordem de nascimento.

Diversos trabalhos têm procurado avaliar a relação entre a ocorrência de partos gemelares e o nível de FSH ( hormônio folículo estimulante). Assim, VOGEL \& MOTULSKY (1986), afirmam que o fato de o nível de FSH aumentar com a idade materna poderia causar uma maior tendência à poliovulação. Essa hipótese foi corroborada pela observação de que mulheres tratadas com hormônios gonadotróficos, por terem ciclos anovulatórios, frequentemente tinham partos gemelares.BORGES-OSÓRIO \& ROBINSON (1993) apontam que o uso de anticoncepcionais orais poderia afetar a frequência de nascimentos gemelares, na medida em que afeta os níveis de FSH. Já segundo MÉTNIK e CZEIZEL (1980), o uso prévio de pílulas anticoncepcionais não afetaria a taxa de gemelaridade.

WHITE \& WYSHAK (1964) apud THOMPSON e cols (1993) sugerem que a tendência à ovulação múltipla que produz gêmeos $D Z$ pode ser vista como um caráter hereditário expressado apenas nas mulheres. Em outras palavras, uma gravidez gemelar é algo causado pela fisiologia da mãe e não pela dos gêmeos. A predisposição genética para a poliovulação relacionada com altos níveis de FSH também é sugerida por BORGESOSÓRIO \& ROBINSON (1993), que indicam para a característica um padrão de herança autossômica limitada ao sexo feminino. Uma evidência favorável seria a proporção de partos gemelares, que diferem significativamente entre gêmeas $D Z(17,1 / 1000)$ e entre esposas de gêmeos $D Z$ (7,2/1000 partos). Além disso, em famílias de gêmeos $D Z$ observa-se que a taxa de nasciments gemelares é alta entre suas irmãs e sobrinhas, porém baixa entre os irmãos. Tal observação favorece a herança autossômica limitada ao sexo feminino, ou, alternativamente uma herança multifatorial com efeito de limiar, sendo o sexo feminino o mais suscetível. 
BORGES-OSÓRIO \& ROBINSON (1993) indicam como fatores que aumentam a frequência de gêmeos $D Z$, o tamanho da irmandade e a incidência nas famílias de indivíduos com aneuploidias, como as síndromes de Down e Turner.

O presente estudo teve por objetivo verificar a frequência de nascimentos gemelares ocorridos no Hospital Universitário de Santa Maria RS (HUSM), no período entre 1985 e 1995. Esse hospital foi escolhido baseado em sua abrangência, já que realiza $o$ atendimento de grande parte da população de Santa Maria e região adjacente. Os dados levantados na presente pesquisa pretende-se que sejam o início de um levantamento amplo sobre a frequência de nascimentos gemelares na cidade de Santa Maria.

\section{MATERIAL E MÉTODOS:}

Foram estudados todos os partos únicos e gemelares ocorridos no Centro Obstétrico (C.O.) do HUSM ,no período entre 19851995. Os dados citados neste estudo, foram retirados dos livros de registro do serviço no C.O. e do arquivo médico do mesmo hospital, (SAME).

Os nascimentos eram contados um a um e anotados por sexo, e quando ocorriam nascimentos múltiplos era feito o registro em formulário próprio. Posteriormente, o registro no SAME da mãe de gêmeos era consultado, obtendo-se aí sua data de nascimento, a fim de calcular a idade da mãe, à época do parto. Os dados foram submetidos ao teste de Weinberg modificado por James $(1979,1984)$ para inferir a proporção MZ/DZ, a partir da distribuição sexual dos gêmeos ao nascimento .Já a razão de sexo foi estimada com base na fórmula proposta por Beiguelman \& Villarroel-Herrera (1992b); onde o número de crianças do sexo masculino por 100 crianças do sexo feminino é obtido pela fórmula $100(2 a+b) /(2 c+b)$, sendo $\mathrm{a}, \mathrm{b}, \mathrm{c}$ os números de pares MM (masculino/masculino), MF (masculino/feminino) e FF (feminino/feminino), respectivamente. Entre os 
partos únicos o número de nascimentos masculinos foi obtido pela relação $100 \mathrm{M} / \mathrm{F}$.

\section{RESULTADOS}

No período 1985 - 1995 ocorreram 16.006 partos, dos quais $253(1,58 \%)$ resultaram de gestações duplas e $06(0,037 \%)$ de gestações triplas, como mostra a tabela 1. A média anual foi de 23 pares de gêmeos e 0,54 de trigêmeos.

Tabela 1 - Tipos de gravidez ocorridos no HUSM no período 1985-1995

\begin{tabular}{cccccc}
\hline \hline ANO & TOTAL & \multicolumn{2}{c}{ GRAVIDEZ DUPLA } & \multicolumn{2}{c}{ GRAVIDEZ TRIPLA } \\
\hline & & $\mathrm{N}$ & $\%$ & $\mathrm{~N}$ & $\%$ \\
\hline 1985 & 1.706 & 20 & 1,17 & --- & --- \\
1986 & 1.758 & 21 & 1,19 & --- & --- \\
1987 & 1.705 & 21 & 1,23 & 01 & 0,058 \\
1988 & 1.521 & 22 & 1,44 & 01 & 0,065 \\
1989 & 1.233 & 20 & 1,62 & -- & -- \\
1990 & 1.181 & 24 & 2,03 & -- & -- \\
1991 & 1.573 & 26 & 1,65 & -- & -- \\
1992 & 1.377 & 29 & 2,10 & 01 & 0,072 \\
1993 & 1.146 & 18 & 1,57 & --- & --- \\
1994 & 1.259 & 22 & 1,74 & 01 & 0,079 \\
1995 & 1.547 & 31 & 2,00 & 02 & 0,129 \\
Total & 16.006 & 254 & 1,61 & 06 & 0,037 \\
\hline
\end{tabular}

A freqüência relativa de gêmeos foi estimada em $44,75 \%$ de pares $D Z$ e 55,25\% de pares $M Z$, analisando-se 249 pares de gêmeos com o sexo determinado. 
Obteve-se nessa amostra 89 pares MM ,52 MF e 108 FF ,cuja razão de sexo foi comparada com a dos partos únicos (7845 do sexo masculino e 7551 do sexo feminino). Foram encontrados, nos nascimentos gemelares 85,82 crianças do sexo masculino por 100 do sexo feminino e, nos partos únicos , 103,89 do sexo masculino por 100 do feminino, resultado esse significativamente diferente pelo teste do qui quadrado $\left(X^{2}=4,39 ; 1 \mathrm{GL}\right.$; $P<0,05)$.

A idade média das mães de crianças gêmeas foi de 27,68 \pm

6,31 anos.

\section{DISCUSSÃO E CONCLUSÕES}

A freqüência de gemelaridade de $1,58 \%$ verificada no HUSM no período estudado, mostrou-se muito semelhante à taxa observada no Rio Grande do Sul, no período de 1992 a 1994 que foi, em média, 1,76\% ( Divisão de Informação em Saúde -DIS- da Secretaria de Saúde e do Meio Ambiente do RS).

A freqüência de $55,25 \%$ de pares $M Z$ e $44,75 \%$ de pares $D Z$ encontrada no presente trabalho difere da obtida por SALDANHA (1980), que relata uma taxa de $1 / 3$ de gêmeos $M Z$, entre caucasóides e negróides. Uma diferença maior a favor dos gêmeos $M Z$ foi verificada por ROLIM e cols.(1995),em um estudo preliminar sobre a população de João Pessoa-PB, onde constataram uma taxa de $29 \%$ de gêmeos $D Z$ e $70,97 \%$ de $M Z$. BEIGUELMAN \& VILLARROEL-HERRERA (1992a) relatam uma diminuição da incidência de gêmeos dizigóticos, na população de Campinas-SP, no período de 1925-1990. Assim, a proporção de pares MZ entre os gêmeos, que era de $30 \%$ na década de 60 , aumentou na atualidade para cerca de $55 \%$, mostrando que agora nascem menos gêmeos DZ (55MZ:45DZ). As causas deste aumento na freqüência de $M Z$ são discutidas pelos autores, o que foi possível devido ao grande período coberto pelo estudo (1925-1990) e ao elevado número de partos analisados (30894), onde foi levado em 
consideração a raça das mães.Tal análise poderia ser feita para a população de Santa Maria, na medida em que se ampliasse a amostra e fosse registrado o grupo racial das mães de gêmeos. Entretando esse dado não aparece na declaração de nascimento do Ministério da Saúde, o que impossibilita uma análise mais acurada da biologia da população em estudo. Quando se considera a razão sexual, vários autores constataram que, ao contrário do que se observa nos recém-nascidos de parto único, a razão de sexo dos gêmeos favorece o sexo feminino. Assim, em um estudo com 1.150 pares de gêmeos de Campinas, São Paulo, BEIGUELMAN \& VILLARROELHERRERA (1992b) observaram uma razão de sexo ao nascer igual a 97,1 do sexo masculino para 100 do sexo feminino ,o que é corroborado pelos dados do presente estudo onde se constatou a razão de sexo nos pares gemelares de 85,82 crianças do sexo masculino para 100 crianças do sexo feminino.

\section{AGRADECIMENTOS}

Os autores agradecem ao Fundo de Incentivo à Pesquisa da UFSM que subvencionou o presente trabalho e concedeu bolsa a Eduardo M. da Costa, e ao HUSM , nas pessoas do Dr. Paulo Afonso Beltrame e da enfermeira Elaine Fuganti, pelo acesso aos registros médicos competentes. Agradecem ainda ao prof. Rocco di Mare pelo auxílio na análise estatística, pelas sugestões e pelo estímulo.

\section{REFERÊNCIAS BIBLIOGRÁFICAS}

BEIGUELMAN,B. Dinâmica dos Genes nas Famílias e nas Populações Ribeirão Preto,Sociedade Brasileira de Genética, 1994. 
BEIGUELMAN, B. \& VILLARROEL-HERRERA, H.O. Investigação dos fatores responsáveis pela diminuição da incidência de gêmeos em populações brasileiras. Rev. Bras. Genet, 15 (sup.1): 167-171, 1992a.

BEIGUELMAN, B. \& VILLARROEL-HERRERA, H.O. Sex ratio of twins in a Southeastern Brazilian population. Rev. Bras. Genet. 15, (3): 707-711, 1992b.

BORGES-OSÓRIO, M.R. \& ROBINSON, W.M. Genética Humana. Porto Alegre, Ed. Universidade UFRGS, Artes Médicas, 1993, 415p.

IMAIZUMI, Y. \& INOUYE, E. Analysis of multiple births in Japan. Acta Genet. Med. Gemellol., 28: 107-124, 1979.

JAMES, W.H. Is Weinberg's differential rule valid? Acta Genet. Med.

Gemellol., 28: 69- 71, 1979.

JAMES, W.H. Twins. N. Engl. J. Med., 311:58, 1984.

McARTHUR,N. Statistics in twin birthies in Italy. Ann.Eugene.17:249, 1953.

MÉTNEKI, J. \& CZEIZEL, A. Contraceptive pills and twins. Acta Genet.

Med. Gemellol., 29: 233-236, 1980.

NYLANDER, P.P.S. The inheritance of DZ twinning. A study of 18.737 maternities in Ibadan, Western Nigéria. Acta Genet.Med. Gemellol., 19: 36-39, 1970.

PARISI, P. \& CAPERNA, G. The changing incidence of twinning: one century of Italian statistics. Em GEDDA, L., PARISI, P. \& NANCE, W.E. (Eds). Twin Research, 3: Twin biology and multiple pregnancy. Alan R. Liss, N. York: 35-48, 1981.

PROPPING,P. \& VOGEL,F. Twin studies in medical genetics. Acta Genet. Med. Gemellol., 25 : 249-258, 1976.

ROLIM, K.L.D.; BEZERRA, V.S.; NUNESMAIA, H.G.S. Gemelaridade na população de João Pessoa- PB. Rev. Bras. Genet. ,18 (3):582, 1995.

SALDANHA. P.H. Gêmeos: hereditariedade versus ambiência. São Paulo: HUCITEC: Ed. da Universidade de São Paulo, 1980. 
THOMPSON, M.W.; McINNERS, R.R. \& WILLARD, H.F. Thompson \& Thompson, Genética Médica, Rio de Janeiro, 5 ed. Guanabara Koogan, 1993, 339p.

VOGEL, F. \& MOTULSKY, A.G. Human Genetics: Problems and Approaches. Berlin, 2 ed. Springer -Verlag, 1986. 
62 Rev. Ciência e Natura, Santa Maria, 19: 53 - 61 ,1997 